\title{
Apoptosis Induction by E2F Decoy DNA of the Prostate Cancer Cell Line
}

\author{
Tao Wang ${ }^{1}$, An -li Jiang ${ }^{2}$, Jian-ye Zhang ${ }^{2 *}$ and Fei Jing ${ }^{3}$ \\ ${ }^{1}$ Institution of Biochemistry; Department of Basic Medicine; Taishan Medical University; YingSheng East Road; \\ 271000; Taian - China. ${ }^{2}$ Institution of Biochemistry and Molecular Biology; Medical School of Shandong \\ University; Jinan. ${ }^{3}$ Taishan Medical University - Taian
}

\begin{abstract}
To observe effect of E2F decoy DNA on proliferation and apoptosis of androgen-independent prostate cancer cell line PC-3M the binding specificity of the E2F decoy DNA to the PC-3M nuclear protein was detected by electrophoretic mobility shift assay (EMSA). E2F decoy DNA, ARE decoy DNA and Control decoy DNA were respectively transfected into PC-3M cells with lipofectamine.Their influence on cell proliferative activity was detected by MTT assay. The cell apoptotic rate was determined by flow cytometric(FCM) analysis and chromosome DNA ladder was detected by DNA gel electrophoresis.The change of mRNA expression of C-myc and CyclinD1 were detected by RT-PCR.The change of $m R N A$ expression of $C$-myc and CyclinDl were detected by Western-blot. EMSA demonstrated specific binding of the E2F decoy to E2F transcription factor.The PC-3M cell growth was inhibited after transfection. The apoptotic rate was $26.35 \%$ and DNA ladder could be observed after transfection.The expression of $C$-myc and CyclinD1 were inhibited. All these results indicated that E2F decoy DNA induced apoptosis of androgen-independent prostate cancer cell lines PC-3M and inhibited cell proliferation via inhibiting expression of $C$-myc and CyclinD1.
\end{abstract}

Key words: Decoy DNA, transcription factors, PC-3M cells, apoptosis

\section{INTRODUCTION}

A series of studies have indicated that E2F expression and activity are involved in proliferation and pathogenesis of tumors. The transcription factor E2F plays a pivotal role in the regulation of cell-cycle and cell proliferation. The seqence elements,which E2F binds to, is present in the promoters of a number of genes that are known and presumed to be important for limiting regulator of DNA synthesis and cell-cycle,protooncogenes,tumor suppressor genes,including DNA polymer $\alpha, P C N A, c-m y c, b-m y b, c d c 2$, cyclinD1, and $\mathrm{Rb}$ and so on(Xin et al,2001; Yamasaki et al, 1996; Kurt et al, 2001). In this study, we synthesized an E2F decoy DNA, a phosphorothiolated double-stranded oligonucleotide which contained the same sequence as the specific DNA sequences (5'TTTNNCGC-3' $\mathrm{N}=\mathrm{C}$ or $\mathrm{G})$ in the promoter of target genes. The E2F decoy DNA was transfected into PC3-M cells to explore the effects of blocking E2F DNA binding domain on growth of PC-3M cells and expression of related genes.

\footnotetext{
* Author for correspondence: wangtaofch@yahoo.com.cn
} 


\section{MATERIALS AND METHODS}

\section{Materials}

All the enzymes were purchased from Takara. RPMI1640 was the product of Hyclone.C-myc antibody CyclinD1 antibody and Western-blotting Luminol Reagent were obtained from Beijing Zhongshang. The human prostate carcinoma cell line PC-3M was provided by our laboratory.

\section{Decoy oligodeoxynucleotides synthesis and annealing}

The E2F decoy was designed according to the E2F elements in the promoter region of the human $\mathrm{C}$ myc gene.the ARE decoy was designed based on the DNA sequence of the ARE in the promoter region of the human PSA gene. The control decoy had three mutations in the consensus E2F element sequences and was used in the control studies. TEN buffer $(10 \mathrm{mmol} / \mathrm{L}$ Tris- $\mathrm{HCl}, \quad 0.1 \mathrm{mmol} / \mathrm{L}$ EDTA, $0.1 \mathrm{~mol} / \mathrm{L} \mathrm{NaCl}, \mathrm{PH}$ 8.0) diluted oligo deoxynucleotides respectively. Complement oligo deoxynucleotides were mixed as equal mol, $95^{\circ} \mathrm{C}$, $10 \mathrm{~min}$, then cooled down to room temperature slowly.

\section{E2F decoy: 5'-TGATTTCCCGCGGAT-3' 3'-ACTAAAGGGCGCCTA-5'}

\section{ARE decoy: \\ 5'-TGCAGAACAGCAAGTGCTAGC-3' 3' -ACGTCTTGTCGTTCACGATCG-5'}

\section{Control decoy: \\ 5'-TGATTACGAGCGGAT-3' 3'-ACTAATGCTCGCCTA-5'}

\section{Electrophoretic mobility shift assay (EMSA)}

The nuclear extracts were prepared from PC-3M cells as decribes (Rita et al, 2002). The protein concentration of nuclear extracts was measured by using Bradford assay. EMSA was performed by using DIG gel shift kit (Roche) according to the manufacture's instruction to confirm specific binding of the E2F decoy to the PC-3M nuclear proteins. Firstly, E2F decoy DNA were labeled with DIG-11-ddUTP at 3'-end by terminal transferase as the probes. 30-60 fmol labeled probes were incubated with the above nuclear extracts $(5-8 \mu \mathrm{g})$ in a buffer for $30 \mathrm{~min}$ at $25^{\circ} \mathrm{C}$. Then, the DNA-protein complexes were separated by electrophoresis using a $6 \%$ native-PAGE at $25 \mathrm{~V}$ for $90 \mathrm{mins}$. The bands in gels were transferred to the positively charged nylon membrane by electroblotting in $0.25 \times \mathrm{TBE}$ transfer buffer at $400 \mathrm{~mA}$ for $40 \mathrm{~min}$. Afterwards, the membrane was baked to fix the bands an incubated with blocking reagent anti-DIG-AP, Fab fragment and CSPD in turn. Finally, the membrane was sealed into hybridization bag and exposed to x-ray film in darkroom. In control experiments, the unlabeled E2F decoy DNA (100 fold and 200 fold molar excess) was added during DNA-protein incubation period. In nonspecific control experiments, the unlabeled control decoy DNA (100 fold molar excess) were used.

\section{Cell cultures}

Human prostate carcinoma cell line PC-3M were cultured in RPMI-1640 medium supplemented with $10 \%$ new born calf serum, $1 \times 105 \mathrm{U} / \mathrm{V}$ penicillin, and $0.1 \mathrm{~g} / \mathrm{L}$ streptomycin at $37{ }^{\circ} \mathrm{C}$ in a $5 \% \mathrm{CO}_{2}$ incubator. Cells between the 37th and 49th generation were used in these experiments. For transfection, cells were grown in phenol redfree and serum free RPMI-1640.

\section{Transfection experiments}

The day before transfection, PC3-M cells were seeded at a density of $2 \times 10^{5} \mathrm{cell} / \mathrm{cm}^{2}$. twenty-four hour later, when cells reached $70-80 \%$ confluency, the decoy ODNs was transfected into PC-3M cells by Lipofectamin ${ }^{\mathrm{TM}} 2000$ according to the manufacturer's instructions. PC-3M cells were divided into four groups. E2F decoy was transfected into one group, ARE decoy was transfected into second group, Control decoy was transfected into third group, the fourth group was used as a internal control. $0.8-1.6 \mu \mathrm{g} / 100 \mu \mathrm{l}$ decoy DNA and $2-4 \mu \mathrm{l} / 100 \mu \mathrm{l}$ lipofectamin were diluted into $100 \mu 1$ medium without serum. Then the above two were combinded and layed at room temperature for $20 \mathrm{mins}$. The complexes were added onto the cells cultured with serum-free medium. 6 hour later, the medium with serum was added.After 24-72h, PC-3M cells were detected.

\section{Cell viability assay}

Measurement of PC-3M cells in vitro proliferation by MTT colorimetric assay. PC-3M cells were seeded at a density of $1 \times 104$ cells/well into 96well plates. MTT $(5 \mathrm{~g} / \mathrm{L})$ was added to the wells $(10 \mu \mathrm{L} /$ well $)$ at 24,48 and $72 \mathrm{~h}$ respectively after transfection as before. After another $4 \mathrm{~h}$ incubation at $37{ }^{\circ} \mathrm{C}$, the medium in the wells were replaced with dimethylsulfoxide(DMSO) $(150 \mu \mathrm{L} /$ well).The plates were agitated at room temperature for 
10min. Absorbance (A value) of every well at $570 \mathrm{~nm}$ was read on an ELISA reader.

\begin{abstract}
Apoptosis assay
Two different assays were used to determine the cell apoptosis.Analysis of DNA fragmentation was carried out as previously described(Barnett et al, 2000).After transfection of 48h, PC-3M cells were harvested and DNA was extracted. The degradation of DNA was investigated by DNA gel electrophoresis.

The apoptotic level of cells incubated with decoy DNA was determined by FACS analysis after PI staining. Approximately $10^{6}$ cells $(70 \%$ confluent monolayer) were transfected with decoy DNA in culture medium without antibiotics at $37^{\circ} \mathrm{C}$ in a $\mathrm{CO}_{2}$ incubator. After transfection of $48 \mathrm{~h}, \mathrm{PC}-3 \mathrm{M}$ cells were harvested and then fixed with ice-cold $70 \%$ ethanol for cytornetric analysis.DNA content of the subdiploid peak, which indicated apoptotic cells, was determined by FACS analysis.
\end{abstract}

Reverse transcriptase-PCR (RT-PCR) Analysis Expression of C-myc gene and CyclinD1 gene was determined by RT-PCR.Total RNA was extracted using TRIzol Reagent(GIBCOBRL). For oligonucleotide treated cells, RNA expression was performed 6 or $24 \mathrm{~h}$ after termination of oligonucleotide treatments. Two mcrogram of total RNA were reverse transcribed using $0.2 \mu \mathrm{g}$ of random hexamer primer and 200 units of murine leukemia virus reverse transcriptase(MBI) under recommended conditions. One-fourth of this cDNA was used as the template in a PCR using the following upstream and downstream primers:C-myc,

\section{5'-CCCAGCGAGGACATCTGGAAGAA-3'}

(upstream primer) and 5'-GAGAAG

CCGCTCCACATGCAGTC-3' (downstream

primer) (expected PCR product,268bp); CyclinD1,

5'-CTGTCGCTGGAGCCCGTGAAAAAG

-3' (upstream primer) and

5'-GAAGTTGTTGGGGCTCCTCAGGT T-

3'(downstream primer) (405bp); $\beta$-actin,

5'-GTGGGGCGCCCAGGCACCAC-3' (upstream

primer) and 5'-CTCCT TA

ATGTCACGCACGATTT-3' (downstream

primer) (500bp). All primers used flank at least one intron.The amplification conditions were denaturation at $94{ }^{\circ} \mathrm{C}$ for $60 \mathrm{sec}$, annealing at $51{ }^{\circ} \mathrm{C}$ for $50 \mathrm{sec}$,extension at $72{ }^{\circ} \mathrm{C}$ for $60 \mathrm{sec}$ for 28 cycles, followed by $10 \mathrm{~min}$ apostextension at $72{ }^{\circ} \mathrm{C}$. Each PCR product was run on an $1.5 \%$ gel electrophoresis at $100 \mathrm{~V}$ for $30 \mathrm{~min}$ and the gel was scanned.

\section{Western blot analysis}

Cell lysates were prepared and Western blots were performed by using total protein, as described previously (Sen-hong et al, 1998).A 85\% confluent 1640 culture flask of PC-3M cells was harvested in a 1:10 solution of trypsin-EDTA and HBSS, centrifuged and resuspended in $350 \mu$ lof $1 \times$ lysis buffer $(5 \times$, premoga), incubated for 20 min, the supernatant recentrifuged and transferred to a fresh tube. An aliquot of $35 \mathrm{ul}$ of the cell lysate was mixed with $30 \mu \mathrm{l}$ of SDS gel loading buffer and resolved by 10\% SDS-PAGE. The gel was transferred to a PDVF membrane and Western blot analysis was performed according to the procedure provided with primary antibody. Briefly, the membrane was incubated at room temperature for $1 \mathrm{~h}$ in a $5 \%$ non-flat milk powder solution in $0.05 \%$ PBS-T (Segma) to block nonspecific reactivity.The blot was then washed three times for 5 min each time with PBS-T alone.It was then incubated for $1 \mathrm{~h}$ at room temperature in PBS$\mathrm{T}$ containing a 1:100 dilution of mouse-reactive cMyc monoclonal antibody (Santa Cruz biotechnology,Inc). After another series of 3-5min washes, the blot was incubated for 45 min with a 1:500 dilution of horseradish peroxdaseconjugated goat anti-mouse secondary antibody (Dako). The blot was again washed three times in PBS-T then once in PBS alone for 5min. Protein detection was performed using ECL chemiluminsescent reagents(Amersham), followed by rapid audtoradiography at room temperature.

\section{Statistical analysis}

All numerical data were expressed as $\mathrm{x} \pm \mathrm{s}$. Results were considered significant, if $\mathrm{P}<0.05$ was obtained by appropriate analysis of variance (ANOVA) procedures and Student's-test.

\section{RESULTS}

\section{Specificity of E2F decoy DNA with protein}

To examine whether the E2F decoy DNA was able to bind to E2F, electrophretic mobility shift assay was performed. The nuclear extracts from PC-3M cells were used for in vitro binding assay with double strands E2F decoy probes and the complex of DNA-protein was observed. In the competition 
study with a 100-fold molar excess of the unlabeled E2F decoy, the formation of this complex was inhibited, though not with the same molar excess of the unlabeled control decoy and E2F decoy, indicating that the binding of the E2F decoy DNA to the PC-3M nuclear protein was specific (Fig. 1).

\section{E2F decoy DNA inhibits proliferation of PC- 3M cells}

When PC-3M cell lines was treated with E2F decoy DNA,ARE decoy DNA and Control decoy DNA and analyzed for viability, the results (Fig. 2) demonstrated that PC-3M cells were quite sensitive to E2F decoy DNA and inhibited while the PC-3M cells were relatively resistant to ARE decoy DNA and Control decoy DNA. E2F decoy DNA had a direct inhibitory effect on the proliferation of $\mathrm{PC}-3 \mathrm{M}$ cells in vitro.
E2F decoy DNA induces apoptosis of PC-3M cells via inhibition of $\mathbf{E} 2 \mathrm{~F}$

Apoptosis was measured after cells were exposed to E2F decoy DNA, ARE decoy DNA and Control decoy DNA for 48h using the DNA fragmentation assay and the FACS analysis. Fig. 3 shows the electrophoretic result of extracted DNA from PC$3 \mathrm{M}$ cells. it clearly indicated DNA degradation after a $48 \mathrm{~h}$ incubation with E2F decoy DNA. No such degradation was observed when PC-3M cells were untreated or incubated with ARE decoy DNA and Control decoy DNA.As shown in Fig. 4 using FACS analysis,the percentage of apoptotic cells after treatment with E2F decoy DNA was increased in PC-3M cells $(26.35 \%)$ compared to that untreated or treated with ARE decoy DNA and Control decoy $\operatorname{DNA}(2.85,2.91$ and $0.40 \%$,respectively).

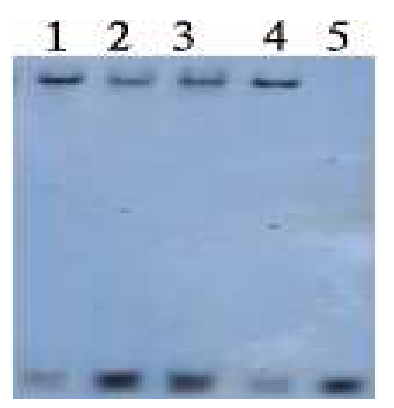

Figure 1 - EMSA of E2F decoy DNA and E2F transcription factor.

1. 4 plus unlabeled Control decoy DNA

2. 4 plus unlabelde E2F decoy DNA

3. 4 plus unlabelde E2F decoy DNA

4. labeled E2F decoy DNA with nuclear extracts

5. labeled E2F decoy DNA

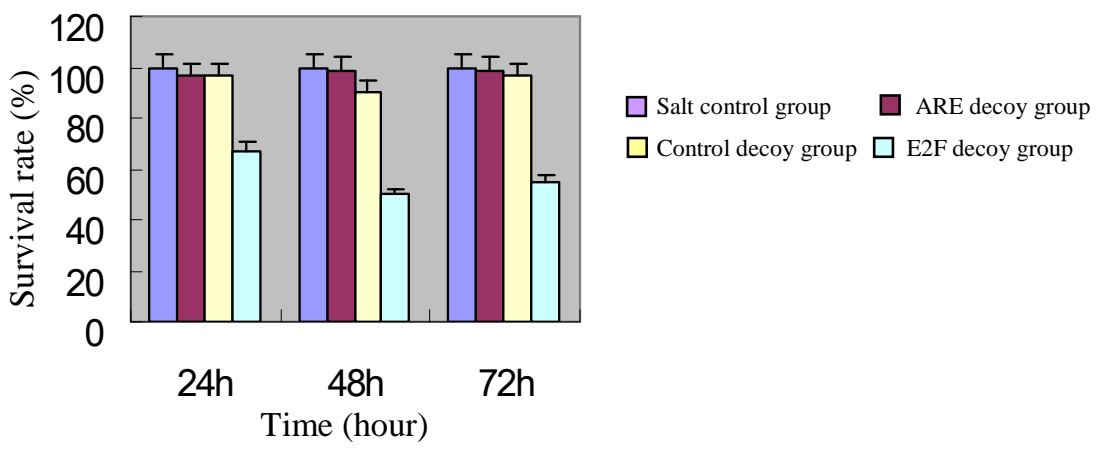

Figure 2 - Cell livability by MTT detection 


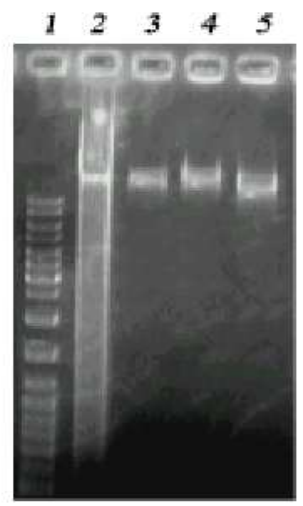

Figure 3 - DNA gel electrophoresis. 1 - Marker 2 - treated with E2F decoy DNA 3 -treated with ARE decoy DNA 4 - treated with control decoy DNA 5 - treated without any decoy DNA.

Decrease of C-myc and CyclinD $D_{1}$ levels Is associated with enhanced apoptosis induced by E2F decoy DNA

To characterize the involvement of E2F decoy DNA in the apoptotic process, we studied an E2Finduced modulation of apoptosis-related molecules such as C-myc, CyclinD ${ }_{1}$.RT-PCR analysis (Figs. 5 and 6) showed a marked decrease of C-myc and CyclinD $_{1}$ mRNA levels in apoptotic cells with E2F decoy DNA with respect to untreated cells and treatment with ARE decoy DNA and Control decoy DNA. The expression of these proteins was also probed by Western blotting with specific antibodies.In fact, an decrease in $\mathrm{C}$-myc and CyclinD $_{1}$ protein expression from PC-3M cells with E2F decoy DNA in Western blot we observed (Figs. 7 and 8). Comparingly no significant changes in $\mathrm{C}$-myc and $\mathrm{CyclinD}_{1}$ expression were observed in these untreated cells and cells treated with ARE decoy DNA and Control decoy DNA.
A

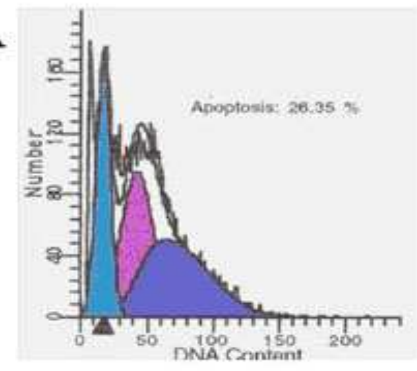

C

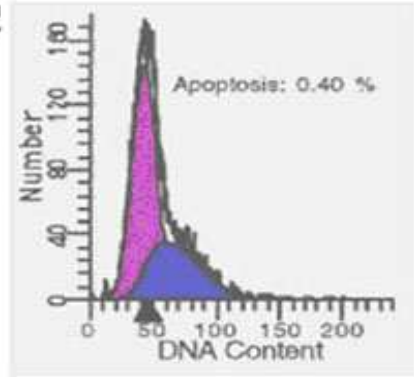

B

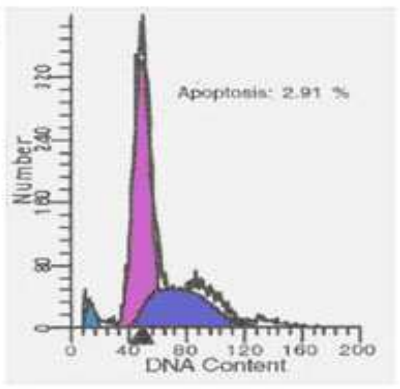

D

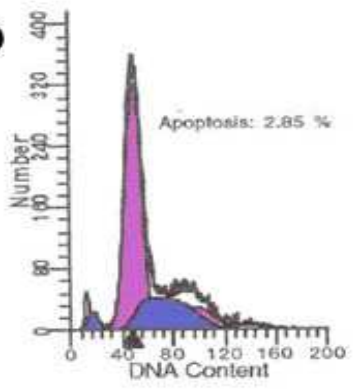

Figure 4 - Apoptotic rate in PC-3M cells treated with. A. E2F decoy DNA. B. ARE decoy DNA. C. control decoy DNA. D. treated without any decoy DNA. 


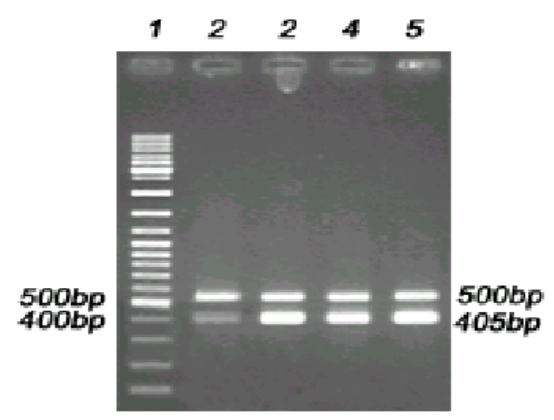

Figure 5 - CyclinD1 expressiong inhibition of E2F decoy DNA in PC-3M cells were analyzed by RT-PCR after 24h of transfection. 1 - Marker 2 - treated with E2F decoy DNA 3 treated with ARE decoy DNA 4 - treated with control decoy DNA 5 -treated without any decoy DNA.

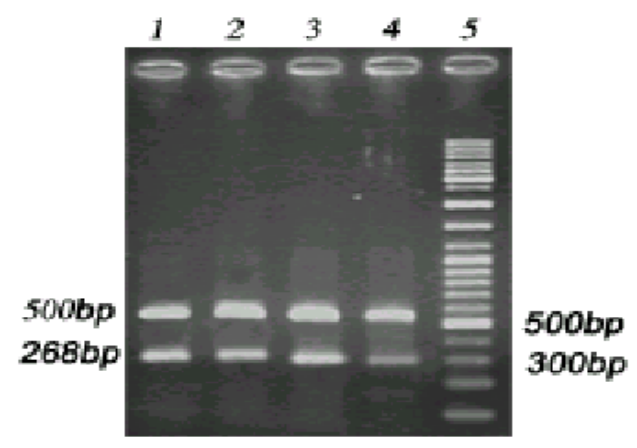

Figure 6 - C-myc expressiong inhibition of E2F decoy DNA in PC-3M cells were analyzed by RT-PCR after 6 h of transfection. 1 - treated without any decoy DNA. 2 - treated with Control decoy DNA. 3 -treated with ARE decoy DNA. 4 - treated with E2F decoy DNA. 5 - Marker.

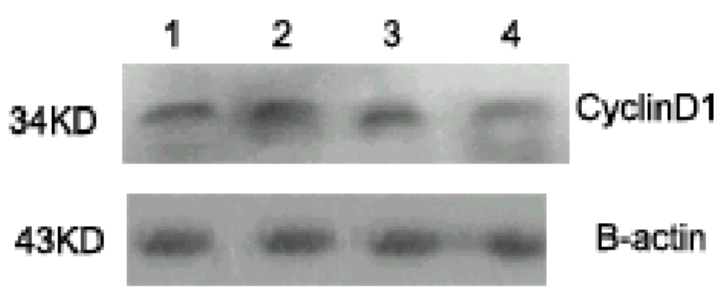

Figure 7 - CyclinD1 expression inhibition of E2F decoy DNA in PC-3M cells were analyzed by Western blotting after $24 \mathrm{~h}$ of transfection. 1- treated without any decoy DNA. 2-.treated with control decoy DNA. 3-treated with ARE decoy DNA. 4-treated with E2F decoy DNA. 


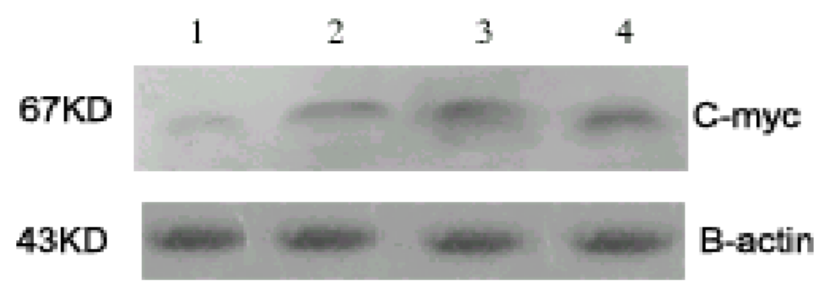

Figure 8 - C-myc expression inhibition of E2F deocy DNA in PC-3M cells were analyzed by Western blotting after $24 \mathrm{~h}$ of transfection. 1-treated with E2F decoy DNA. 2-ARE decoy DNA. 3control decoy DNA. 4-treated without any deocy DNA.

\section{DISCUSSION}

E2F transcription factor plays an important role in progression into S-phase and that this probably coincides with capacity to stimulate transcription. Lines of evidence implicate the E2F transcription factor as an important component of cell proliferation control. First, E2F binding sites are found in the promoters of genes responsive to proliferation signals and the level of E2F binding activity increases at a time when many of these genes are activated. Second, the tumour suppressor protein $\mathrm{Rb}$, as well as the related p107 protein,complexes with $\mathrm{E} 2 \mathrm{~F}$, resulting in an inhibition of E2F transcriptional activity.Third, oncogenic products of the DNA tumour viruses can dissociate these E2F complexes.

Transfection of decoy ODNs has been reported to be a powerful therapeutic strategy against hyperplasia $\square$ acute and chronic inflammation and tumour (Morishita et al, 1998; Tomita et al, 2003; Yamasaki et al, 2003). transfection of doublestranded ODNs corresponding to the cis sequence will result in the attenuation of authentic cis-trans interaction, leading to the removal of transcription factors from the endogenous cis-element and the suppression of the expression of regulated genes. In this experiments, we synthesized double-strands oligodeoxynucleotides (ODNs), which had consensus sequence with endogenous E2F ciselement and high-affinity to the E2F transcription factor. E2F decoy DNA was transfected into androgen-independent prostate cancer cells line PC-3M by which induction of apoptosis was demonstrated and competed with the endogenous E2F cis-element for binding to the E2F transcription factor. In conclusion, E2F decoy DNA coud inhibit proliferation of PC-3M cell and induced the apoptosis of PC-3M cells through binding the E2F competitively and inhibiting the function of $\mathrm{E} 2 \mathrm{~F}$ on expression of $\mathrm{C}$-myc and CyclinD $_{1}$. Decoy DNA strategy could provide a new method for therapy of prostate cancer.

\section{ACKNOWLEDGMENTS}

This research was supported by the National Natural Science Foundation of China (NO.30470820). We thank to Dr. Yong Gang Song and Dr. Qian Liu, for suggestions and revision, to Dr.Peng Ju Zhang for providing the E2F decoy DNA.

\section{REFERENCES}

Barnett, F. D.; Abul-Milh, M.; Huesca M, Lingwood C A. (2000) Enterohemorrhagic Escherichia coli induces apoptosis which augments bacterial binding and phosphatidylethanolamine exposure on the plasma membrane outer leaflet[J]. Infect Immun.; 68, 3108-3115.

Kurt, H. et al. (2001), E2F Activity Is Biphasically Regulated by Androgens in LNCaP Cells $[\mathrm{J}]$.Biochemical and Biophysical Research Communications. 283, 97-101.

Morishita, R.; Higaki J. et al. (1998), Application of transcription factor 'decoy' strategy as means of gene therapy and study of gene expression in cardiovascular disease [J]. Circ Res, 82 (10),10231028.

Rita, C.; Marzia, B. Lucia G. et al. (2002), Design and characterization of decoy oligonucleotides containing locked nucleic acids[J].Nucleic Acids Research. 30(11), 2435-2443. 
Sen-hong, Z.; Kerry, L. (1998), Antiproliferative effect of 1 $\alpha, 25$-dihydroxyvitamin $\mathrm{D}_{3}$ in human prostate cancer cell line LNCaP involves reduction of Cyclindependent kinase2 activity and persistent G1 accumulation[J].Endocrinology. 139(3), 1197-1207.

Tomita, N.; Azuma, H.; Kaneda, Y. et al. (2003), Gene therapy with transcription factor decoy oligonucleotides as a potential treatment for cardiovascular diseases[J]. CurrDrug Targets, 4(4), 339-346.

Xin, Z.; Mark, L. (2001), Rb activation and repression of C-MYC transcription precede apoptosis of human prostate epithelial cells [J].Urology. 57, 860.
Yamasaki, L. et al. (1996), Tumor induction and tissue atrophy in mice lacking E2F[J].Cell. 85, 537-548.

Yamasaki, K.; Asai, T.; Shimizu, M. et al. (2003), Inhibition of NF-kappa B activation using ciselement'decoy' of NF-kappa B binding site reduces neointimal formation in porcineballoon- injured coronary artery model [J]. Gene Ther. 10(4), 356364.
Received: May 19, 2007; Revised: November 03, 2007; Accepted: October 02, 2009. 